MITSUBISHI ELECTRIC RESEARCH LABORATORIES

http://www.merl.com

\title{
Overview of the Emerging HEVC Screen Content Coding Extension
}

\author{
Xu, J.; Joshi, R.; Cohen, R.A. \\ TR2015-126 September 2015
}

\begin{abstract}
A Screen Content coding (SCC) extension to High Efficiency Video Coding (HEVC) is currently under development by the Joint Collaborative Team on Video Coding (JCT-VC), which is a joint effort from the ITU-T Video Coding Experts Group and the ISO/IEC Moving Picture Experts Group. The main goal of the HEVC screen content coding standardization effort is to enable significantly improved compression performance for videos containing a substantial amount of still or moving rendered graphics, text, and animation rather than, or in addition to, camera-captured content. This paper provides an overview of the technical features and characteristics of the current HEVCSCC test model and related coding tools, including intra block copy, palette mode, adaptive colour transform, and adaptive motion vector resolution. The performance of the screen content coding extension is compared against existing standards in terms of bit-rate savings at equal distortion.
\end{abstract}

2015 IEEE Transactions on Circuits and Systems for Video Technology

This work may not be copied or reproduced in whole or in part for any commercial purpose. Permission to copy in
whole or in part without payment of fee is granted for nonprofit educational and research purposes provided that all
such whole or partial copies include the following: a notice that such copying is by permission of Mitsubishi Electric
Research Laboratories, Inc.; an acknowledgment of the authors and individual contributions to the work; and all
applicable portions of the copyright notice. Copying, reproduction, or republishing for any other purpose shall require
a license with payment of fee to Mitsubishi Electric Research Laboratories, Inc. All rights reserved.

Copyright (c) Mitsubishi Electric Research Laboratories, Inc., 2015

201 Broadway, Cambridge, Massachusetts 02139 



\title{
Overview of the Emerging HEVC Screen Content Coding Extension
}

\author{
Jizheng Xu, Senior Member, IEEE, Rajan Joshi, Member, IEEE, and Robert A. Cohen, Senior Member, IEEE
}

\begin{abstract}
A Screen Content coding (SCC) extension to High Efficiency Video Coding (HEVC) is currently under development by the Joint Collaborative Team on Video Coding (JCT-VC), which is a joint effort from the ITU-T Video Coding Experts Group and the ISO/IEC Moving Picture Experts Group. The main goal of the HEVC screen content coding standardization effort is to enable significantly improved compression performance for videos containing a substantial amount of still or moving rendered graphics, text, and animation rather than, or in addition to, camera-captured content. This paper provides an overview of the technical features and characteristics of the current HEVCSCC test model and related coding tools, including intra block copy, palette mode, adaptive colour transform, and adaptive motion vector resolution. The performance of the screen content coding extension is compared against existing standards in terms of bit-rate savings at equal distortion.
\end{abstract}

Index Terms-HEVC, video coding, screen content coding.

\section{INTRODUCTION}

$\mathbf{I}$ N January 2013, the first edition of the High Efficiency Video Coding standard, also known as HEVC version 1 [1], was finalized. This work was done by the Joint Collaborative Team on Video Coding (JCT-VC), which was established jointly in 2010 by ITU-T Study Group 16 (VCEG) and ISO/IEC JTC1/SC 29/WG 11 (MPEG). One of the primary requirements of that standard was that it demonstrates a substantial bit-rate reduction over the existing H.264/AVC [2] standard. Both the initial development of HEVC and the earlier development of H.264/AVC focused on compressing camera-captured video sequences. Although several different test sequences were used during the development of these standards, the camera-captured sequences exhibited common characteristics such as the presence of sensor noise and an abundance of translational motion. Recently, however, there has been a proliferation of applications that use video devices to display more than just camera-captured content. These applications include displays that combine camera-captured and computer graphics, wireless displays, tablets, automotive displays, screen-sharing, etc. [3]. The type of video content used in these applications can contain a significant amount of stationary or moving computer graphics and text, along with camera-captured content. However, unlike camera-captured content, screen content frequently contains no sensor noise, and such content may have large uniformly flat areas, repeated

Copyright (c) 2015 IEEE. Personal use of this material is permitted. However, permission to use this material for any other purposes must be obtained from the IEEE by sending an email to pubs-permissions@ieee.org

$\mathrm{J}$. Xu is with Microsoft Research, Beijing 100080, China.

R. Joshi is with Qualcomm Technologies, Inc.

R. A. Cohen is with Mitsubishi Electric Research Laboratories. patterns, highly saturated or a limited number of different colours, and numerically identical blocks or regions among a sequence of pictures. These characteristics, if properly leveraged, can offer opportunities for significant improvements in compression efficiency over a coding system designed primarily for camera-captured content.

During the development of HEVC, decisions as to which tools to incorporate into the version 1 standard were made primarily based on their coding performance on cameracaptured content. Several tool proposals showed that the characteristics of screen-content video can be leveraged to obtain additional improvements in compression efficiency over the HEVC version 1 standard under development.

Residual Scalar Quantization (RSQ) and Base Colors and Index Map (BCIM) [4] were proposed early during the HEVC development process. Because screen content often has high contrast and sharp edges, RSQ directly quantized the intra prediction residual, without applying a transform. BCIM took advantage of the observation that the number of unique colours in screen content pictures is usually limited as compared to camera-captured content. RSQ and BCIM could respectively be considered early forms of transform skip, which is part of HEVC version 1, and palette mode, which is described in Section III-B. Additional modes such as transform bypass where both the transform and quantization steps are bypassed for lossless coding, and the use of differential pulse code modulation (DPCM) for sample-based intra prediction were proposed in [5].

Because screen content often contains repeated patterns, dictionary and Lempel-Ziv coding tools were shown to be effective at improving coding efficiency, especially on pictures containing text and line graphics [6], [7], [8]. These tools store a dictionary of previously-coded samples. If a block or coding unit contains strings of samples that are already contained in the dictionary, then a pointer to the dictionary can be signalled, thus avoiding the need to transform and quantize a prediction residual.

In January 2014, the MPEG Requirements subgroup published a set of requirements for an extension of HEVC for coding of screen content [3]. This document identified three types of screen content: mixed content, text and graphics with motion, and animation. Up to visually lossless coding performance was specified, for RGB and YUV 4:4:4 video having 8 or 10 bits per colour component. Given the earlier published drafts of these requirements, along with the existing evidence that additional tools could improve the coding performance of HEVC on screen content material, VCEG and MPEG issued a joint Call for Proposals (CfP) for coding of screen content [9]. 
At the next JCT-VC meeting in March 2014, seven responses to the CfP were evaluated. After identifying the tools that produced significant improvements in performance for screen content, several core experiments (CEs) were defined. The tools included in these CEs were: intra block copying extensions, line-based intra copy, palette mode, string matching for sample coding, and cross-component prediction and adaptive colour transforms. After evaluating the outcome of the CEs and related proposals, the HEVC Screen Content Coding Draft Text 1 [10] was published in July 2014.

This paper gives an overview of the tools and coding performance associated with this new HEVC screen content coding extension. At the time of this writing, the current version of the text is Draft 2 [11]. Section II describes screen content coding support in HEVC version 1 and HEVC range extensions. New coding tools in the HEVC screen content coding extension (HEVC-SCC) are described Section III. Section IV presents new encoding algorithms introduced during the development of HEVC-SCC. Coding performance comparisons are discussed in Section V, and Section VI concludes the paper.

\section{SCREEN CONTENT CODING SUPPORT IN HEVC}

The HEVC screen content coding extension (HEVC-SCC) is developed based on HEVC version 1 [1] and HEVC range extensions (HEVC-RExt) [12]. Thus, it inherits the coding structure and coding tools of HEVC version 1 and HEVCRExt. HEVC-SCC also maintains backward compatibility to HEVC version 1 and HEVC-RExt. As an example, an HEVCSCC decoder can decode HEVC version 1 bit-streams and the decoded videos are identical to those produced by an HEVC version 1 decoder. Screen content, as one class of video, was also considered during the development of HEVC version 1 and HEVC-RExt, although it was not the main focus of those standards. In the following subsections, the structure of HEVC and HEVC-RExt, along with the coding tools that primarily targeted screen content, are briefly introduced.

\section{A. HEVC version 1}

HEVC version 1 follows the conventional hybrid coding structure as in previous video coding standards. An input image is first divided into image blocks, referred to as coding tree units (CTU), of pre-defined size. The size of a CTU can be of $16 \times 16,32 \times 32$, or $64 \times 64$ luma samples. A CTU contains corresponding chroma samples according to the input colour format. A quad-tree split, with a CTU as the root, divides the CTU into one or more coding units (CU). A CU is square-shaped and can have $8 \times 8,16 \times 16,32 \times 32$, or $64 \times 64$ luma samples and corresponding luma samples. A CU can be classified as an intra $\mathrm{CU}$ or an inter $\mathrm{CU}$, and it is further divided into one, two or four prediction units (PU). For an intra $\mathrm{CU}$, spatially neighboring reconstructed samples are used to predict its PUs. For an inter CU, a motion vector is sent for each PU, which is used by a motion compensation process to generate the prediction from other pictures.

A transform quad-tree is also defined for a CU to indicate how the predicted residual are decorrelated with spatial transforms after the prediction process. A leaf of a transform quad-tree is referred to as a transform unit (TU). An integer transform is applied to each TU. All transforms applied to an inter $\mathrm{CU}$ and most transforms applied to an intra $\mathrm{CU}$ are derived to approximate the discrete cosine transform (DCT) of appropriate size. The only exception is the $4 \times 4$ transform applied to an intra $\mathrm{CU}$, which is designed to approximate the discrete sine transform (DST).

During the development of HEVC version 1, it was observed that for screen content, spatially transforms mentioned above do not always help in improving coding efficiency. For most screen content, the images are sharp, containing irregular edges and shapes. After the prediction process, the residual signal may already be sparse because the background can be precisely predicted while irregular foreground may not. In such a case, the existing transform will spread the energy to most frequencies instead of compacting the energy, thereby destroying the sparsity of the residual, leading to low coding efficiency during entropy coding. Thus, for those blocks, skipping the transform and quantizing data in the spatial domain can be a better choice, as was demonstrated for H.264/AVC in [13]. HEVC version 1 can skip the transform for a $4 \times 4 \mathrm{TU}$, whether it is intra [14] or inter [15]. This transform skip is equivalent to applying an identity transform to the TU. Thus, the quantization process after applying transform skip is the same as that applied after the spatial transform. It turns out that such a simple design can lead to significant coding efficiency improvement for screen content, e.g. the bit-saving brought by the transform skip mode is about $7.5 \%$ for typical $4: 2: 0$ screen content [15]. When applied to $4: 4: 4$ screen content, the coding gain for transform skip is much larger [16], ranging from $5.5 \%$ to $34.8 \%$.

\section{B. HEVC-RExt}

After HEVC version 1, HEVC-RExt was developed to support non-4:2:0 colour formats, e.g. 4:4:4 and 4:2:2v, and high bit-depth video, e.g. up to 16-bit. Because most screen content is captured in the 4:4:4 colour format, which is not supported by HEVC version 1, more attention was given to coding of screen content in HEVC-RExt. The coding tools that improved the coding efficiency for screen content in HEVCRExt compared with HEVC version 1 include:

1) Improvements to transform skip mode: As mentioned above, HEVC version 1 only supports transform skip for $4 \times 4$ TUs. HEVC-RExt extends transform skip to all TUs, regardless of their size [17]. Enabling transform skip for all TUs has two benefits. One is that the coding efficiency for screen content can be further improved. The other is that encoders have the flexibility to exploit the transform skip mode. For example, a specific encoder may support only large transform units so that the encoding complexity can be reduced. If transform skip is allowed only for $4 \times 4 \mathrm{TUs}$, the performance of such an encoder would be affected adversely since it cannot exploit the benefit brought by transform skip, which can be much more noticeable for screen content. Other improvements include coefficient coding for transform skip blocks. For an intra transform block, when a spatial transform is applied to the residual, the energy is compacted at the upper-left corner of the 
block. However, when transform skip is used on a transform block, because its prediction is from the adjacent upper row and/or left column of samples, the prediction error is higher at the bottom-right corner compared to the upper-left corner. The different characteristics of the residual of transform skip blocks relative to transformed blocks make entropy coding designed for transformed blocks inefficient when applied to transform skip blocks. A simple yet effective solution adopted in HEVC-RExt is to rotate the residual of transform skip blocks by 180 degrees [18], [19]. This rotation process is only applied to $4 \times 4$ intra transform skip blocks [20]. Another improvement for the entropy coding of the transform skip blocks is that a single context is used to code the significant coefficient map. For transformed blocks, the context used for significance coding depends on the position (frequency) of the coefficient. However, since all the coefficients of a transform skip block are from the residual in the spatial domain, using a single context is more reasonable that using different contexts depending on position.

2) Residual differential pulse code modulation (RDPCM): Even after intra prediction, there is still correlation in the residual signal which can be exploited. Residual differential pulse code modulation (RDPCM) predicts the current residual using its immediately neighboring residual. In HEVC-RExt, RDPCM was proposed for intra lossless coding [21]. Then it was extended to lossy coding [22] and inter coding [23]. In RDPCM, the left reconstructed residual or the above one is used to predict the current residual, depending on whether the block uses horizontal or vertical RDPCM. RDPCM is used only for transform skip blocks. In inter mode, flags are sent to indicate whether RDPCM is used and if so, its direction. In contrast, RDPCM in intra mode is applied in the same direction as the intra prediction direction. Because of this, using the reconstructed residual to predict the current residual is identical to using reconstructed samples to predict the current sample in intra mode (if clipping of the reconstructed sample is ignored). From this perspective, RDPCM shortens the prediction distance, leading to better prediction. For screen content, a short distance prediction is quite useful because usually the content is sharp and changes rapidly.

3) Cross-component prediction (CCP): CCP [24] and its predecessor LM Chroma mode [25] were proposed to exploit correlation among colour components [24]. In CCP, the residual of the second or third colour component can be predicted from the residual of the first colour component multiplied by a scaling factor. The factor is selected from $\left\{0, \pm \frac{1}{8}, \pm \frac{1}{4}, \pm \frac{1}{2}, \pm 1\right\}$ and is signalled to the decoder. One advantage of performing prediction on residuals in the spatial domain is that the reconstruction process for the second and third colour components does not depend on reconstructed samples of the first colour component. Hence the reconstruction process for different colour components can be performed in parallel once the residuals for the three colour components are available. Although such a design can still leave a certain degree of correlation among colour components, it was demonstrated that CCP can significantly improve the coding efficiency when coding videos having the RGB colour format. Because much screen content is captured in the RGB domain,
CCP is very effective in coding of screen content.

4) Other improvements: Some other aspects of HEVCRExt, although not specifically designed for screen content coding, also improve the coding efficiency for screen content. For example, the initialization of Rice parameters based on previous similar blocks was primarily designed for high bitdepth coding; but it also showed improvement for coding screen content [26].

\section{HEVC-SCC}

Unlike HEVC version 1 and HEVC-RExt, the tools added for the HEVC-SCC extension focus primarily on coding screen content. To illustrate the framework of SCC, an SCC encoder is shown Fig. 1. As shown in the figure, HEVC-SCC is based on the HEVC framework while several new modules/tools are added. The new coding tools are:

- Intra block copy (IBC): HEVC-SCC introduces a new $\mathrm{CU}$ mode in addition to the conventional intra and inter modes, referred to as intra block copy (IBC). When a CU is coded in IBC mode, the PUs of this CU find similar reconstructed blocks within the same picture. IBC can be considered as "motion compensation" within the current picture.

- Palette mode: For screen content, it is observed that for many blocks, a limited number of different colour values may exist. Thus, palette mode enumerates those colour values and then for each sample, sends an index to indicate to which colour it belongs. Palette mode can be more efficient than the prediction-then-transform representation.

- Adaptive colour transform (ACT): Because much screen content uses the RGB colour space, removing inter-colour component redundancy is important for efficient coding. Earlier work with adaptive colour space transforms was shown to yield improvements in coding efficiency [27]. In HEVC-SCC, a CU-level adaptation is used to convert residual to different colour spaces. More precisely, an image block in the RGB colour space can be coded directly. Or it can be converted adaptively to the $\mathrm{YCoCg}$ colour space during coding.

- Adaptive motion vector resolution: Unlike cameracaptured content, where motion is continuous, screen content often has discrete motion, which has a granularity of one or more samples. Thus, for much screen content, it is not necessary to use fractional motion compensation. In HEVC-SCC, a slice-level control is enabled to switch the motion vectors between full-pel and fractional resolutions.

The details of these new tools are described in the following sections.

\section{New Coding TOOLS IN HEVC-SCC}

This section describes the new coding tools that were adopted into the HEVC-SCC text specifications, i.e. normative specifications. When relevant, some non-normative aspects are included in this section, and a more complete description of non-normative aspects of the tools is included in the section on encoding algorithms. 


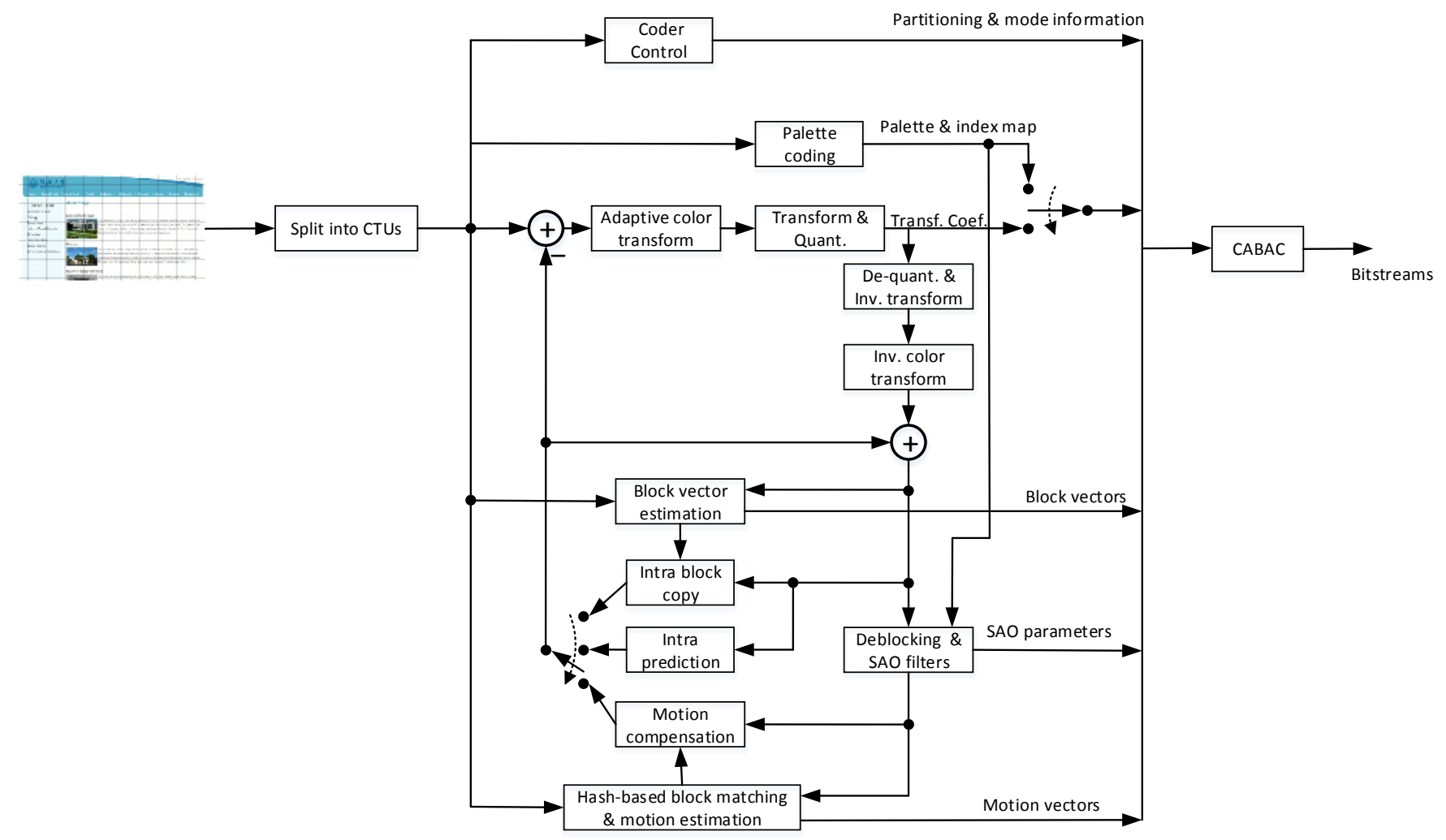

Fig. 1. Encoder for screen content coding extension.

\section{A. Intra Block Copy}

Intra Block Copy (IBC) is a coding tool similar to interpicture prediction. The main difference is that in IBC, a predictor block is formed from the reconstructed samples (before application of in-loop filtering) of the current picture. Previously, IBC was proposed in the context of AVC/H.264 [28] but the coding gain was not consistently high across different test sequences, which at the time were primarily camera-captured sequences and not screen content material. A CU based IBC mode was proposed during the HEVC-RExt development [29]. A modified version [30] was adopted into the HEVC-RExt text specification but was later removed. IBC has been a part of HEVC-SCC test model since the beginning of the HEVC-SCC development.

At the early stage of HEVC-SCC development, IBC was performed at the coding unit (CU) level. A block vector was coded to specify the location of the predictor block. However, since both IBC and inter mode share the concept of vectors representing displaced blocks, it is natural to unify the design of IBC and inter mode. Methods to unify these modes [31], [32] have shown that also using the inter mode syntax design for IBC is an adequate choice.

In the current HEVC-SCC design, IBC is performed at the prediction unit (PU) level and is treated as an inter PU. Specifically, using the inter mode design, the current picture can also be used as a reference picture for IBC. When a PU's reference picture is the current picture, it means that its prediction is performed from the reconstructed samples, before in-loop filtering in the encoder, of the current picture, which corresponds to the original IBC design. When the current picture is used as a reference, it is marked as a longterm reference. After the current picture is fully decoded, the reconstructed picture after in-loop filtering is added to the decoded picture buffer (DPB) as a short-term reference, which is identical to what HEVC version 1 does after decoding a picture. Using the inter mode design to enable IBC at the PU level enables greater flexibility in combining IBC and inter mode. For example, an inter CU can have two PUs, one using conventional inter mode and the other using IBC; a PU can be bidirectionally predicted from an average between a block from the current picture and a block from a previous picture.

However, unification between IBC and inter mode does not mean that IBC can be directly implemented as an inter mode in practice. The implementation of IBC can be much different from that of inter mode in many platforms. Such differences exist because when the current picture is used as a reference, it has not been fully reconstructed, whereas the other reference pictures have been decoded and stored in the DPB.

For IBC mode, because the block to be processed and its prediction are from the same picture, several constraints have been placed to avoid affecting other modules adversely. The constraints for IBC mode are:

- The predictor block may not overlap the current $\mathrm{CU}$, to avoid generating predictions from unreconstructed samples.

- The predictor block and the current CU should be within the same slice and the same tile. Otherwise, there will be dependency among different slices or tiles, which affect 


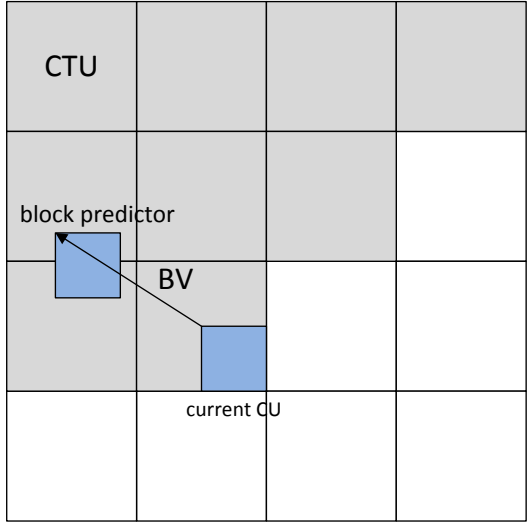

Fig. 2. Search area for IBC (shown in gray).

the parallel processing capability provided by the design of slices and tiles.

- The predictor block is normatively required to be entirely contained in the search region shown in Fig. 2. It is so designed to avoid affecting the parallel processing capability provided by wavefronts. To simply the design, the constraint still holds even when wavefronts are not being used.

- For constrained-intra profiles, the samples of the predictor block must be from other intra blocks or IBC blocks.

- The block vector precision is full-pel.

\section{B. Palette mode}

Palettes are an efficient method for representing blocks containing a small number of distinct colour values. Rather than apply a prediction and transform to a block, palette mode signals indices to indicate the colour values of each sample. An early use of palettes was in the conversion of 24bit RGB images to 8-bit index images to save on RAM or video memory buffer space. A CU based palette coding mode was proposed during the HEVC-RExt development [33]. The palette mode was further refined through core experiments and Ad Hoc Group (AHG) discussions and was adopted [34] into the SCC text specification.

A palette refers to a table consisting of representative colour values from the $\mathrm{CU}$ coded using the palette mode. For each sample in the $\mathrm{CU}$, an index into the current table is signalled in the bit-stream. The decoder uses the palette table and the index to reconstruct each sample of the CU. Each entry in the palette table consists of three components (RGB or $\mathrm{YCbCr}$ ). For 4:2:0 and 4:2:2 colour formats, if no chroma components are present for the current sample position, only the first component is used for reconstruction. A special index, known as an escape index, is reserved to indicate that a sample does not belong to the palette. In such a case, in addition to coding the escape index, the quantized values of the component(s) of the escape sample are also coded in the bit-stream.

The size of the palette table is referred to as the palette size. If the palette size is nonzero, then the indices from zero to palette size minus one are reserved for denoting entries from the palette, and the escape index is set equal to the palette

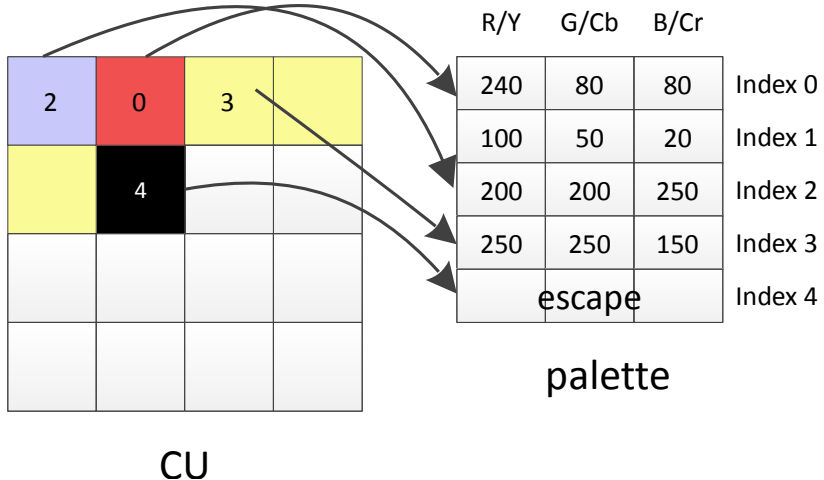

Fig. 3. Palette example (palette size $=4$ ).

size. An example for this coding is illustrated in Fig. 3. On the encoder side, palette table derivation is performed. This is discussed further in Section IV-C. Normative aspects of palette coding can be divided into two categories: coding of the palette table and coding of the palette indices of the samples in the CU.

The palette needs to be signalled to the decoder. It is typical that a palette shares some entries with neighboring blocks that are coded using the palette mode. To exploit this sharing, the palette table is coded using a combination of prediction from palette entries of previously coded CUs and new palette entries that are explicitly signalled. For prediction, a predictor palette consisting of palette entries from the previous CUs coded in palette mode is maintained. For each entry in the palette predictor, a flag is signalled to specify whether that entry is reused in the current palette. The collection of flags is signalled using run-length coding of zeros. The run-lengths are coded using exponential Golomb code of order 0. After signalling the predicted palette entries, the number of new palette entries and their values are signalled. This is illustrated in Fig. 4. In this example, the predictor palette contains six entries. Out of these, three are reused in the current palette (0th, 2nd and 3rd). These are assigned indices 0,1 and 2, respectively, in the current palette. This is followed by 2 new entries.

For the first CTU of each slice, tile and CTU row (when wavefronts are used), the palette predictor is initialized using initialization entries signalled in the picture parameter set (PPS) or to zero. After a CU has been coded in palette mode, the palette predictor is updated as follows. First all the entries from the current palette are included in the predictor palette. This is followed by all the entries that were not reused from the previous predictor palette. This process is continued till all the entries from the previous predictor palette that were not reused are included or the maximum palette predictor size is reached. The updated predictor palette is illustrated on the right side in Fig. 4.

To code the palette indices, first a flag, palette_escape_val_present_flag, is coded to indicate whether there are any escape indices present in the current CU. Two different scans may be used to code the palette indices in a CU, namely, horizontal traverse scan and vertical traverse scan as shown in Fig. 5. In the following description, a 


\begin{tabular}{|c|c|c|c|c|c|c|c|c|}
\hline \multicolumn{4}{|c|}{ predictor palette } & \multicolumn{5}{|c|}{ current palette } \\
\hline Index & $\mathrm{G} / \mathrm{Y}$ & $\mathrm{B} / \mathrm{Cb}$ & $\mathrm{R} / \mathrm{Cr}$ & Reuse flag & Index & $\mathrm{G} / \mathrm{Y}$ & $\mathrm{B} / \mathrm{Cb}$ & $\mathrm{R} / \mathrm{Cr}$ \\
\hline 0 & G0 & BO & Ro & 1 & 0 & G0 & BO & Ro \\
\hline 1 & G1 & B1 & R1 & 0 & & & & \\
\hline 2 & $\mathrm{G} 2$ & B2 & R2 & 1 & 1 & G2 & B2 & R2 \\
\hline 3 & G3 & B3 & R3 & 1 & 2 & G3 & B3 & R3 \\
\hline 4 & G4 & B4 & R4 & 0 & & & & \\
\hline 5 & G5 & B5 & R5 & 0 & & & & \\
\hline & & & & & 3 & G3N & B3N & $\mathrm{R} 3 \mathrm{~N}$ \\
\hline & & & pare & & 4 & G4N & B4N & $\mathrm{R} 4 \mathrm{~N}$ \\
\hline
\end{tabular}

Updated predictor palette
\begin{tabular}{|c|c|c|c|}
\hline Index & G/Y & B/Cb & R/Cr \\
\hline 0 & G0 & B0 & R0 \\
\hline 1 & G2 & B2 & R2 \\
\hline 2 & G3 & B3 & R3 \\
\hline 3 & G3N & B3N & R3N \\
\hline 4 & G4N & B4N & R4N \\
\hline 5 & G1 & B1 & R1 \\
\hline 6 & G4 & B4 & R4 \\
\hline 7 & G5 & B5 & R5 \\
\hline
\end{tabular}

Fig. 4. Construction of palette from predictor palette and new explicitly signalled entries.

horizontal traverse scan is assumed. If the scan is vertical traverse, the CU index map may be transposed before coding (or after decoding). The direction of the scan is signalled using palette_transpose_flag.

Since screen content may typically contain flat areas with uniform or near-uniform sample values, run-length coding of palette indices is an efficient method of compression. Additionally, it is observed that indices in consecutive rows or columns may be identical. To exploit this, each palette sample may be coded in one of two palette sample modes, COPY_INDEX_MODE and COPY_ABOVE_MODE, which are signalled in the bitstream as palette_run_type_flag. In COPY_INDEX_MODE, the palette index is coded followed by a run value which specifies the number of subsequent samples that have the same index. The index values are coded using a truncated binary code [35]. In COPY_ABOVE_MODE, the palette index is copied from the previous row. This is followed by a run value which specifies the number of subsequent positions for which the index is copied from the previous row. Both COPY_INDEX_MODE and COPY_ABOVE_MODE runs may span multiple rows. In COPY_ABOVE_MODE only the run value is signalled but not the index. If the index for a particular sample corresponds to the escape index, the component value(s) are quantized and coded. Fig. 6 shows an example of palette sample modes, indices and run values for a $4 \times 4$ block $^{1}$.

The run coding uses a concatenation of unary code and exponential Golomb code of order zero. The code can be described as follows. A run of zero is represented as "0". A run of length $L \geq 1$ is represented as a concatenation of "1" and the exponential Golomb code (order zero) representation for $(L-1)$. Both prefix and suffix are truncated based on the maximum possible run value when the run continues to the end of the block. The code is specified in Table I.

Several redundancies are exploited in the coding of palette indices to reduce the number of syntax elements and make the coding more efficient. For example, when the palette size is equal to 0 or when the palette size is equal to 1 and there are no escape indices present, then the in-

\footnotetext{
${ }^{1}$ The $4 \times 4$ block is chosen for convenience. The minimum palette block size is $8 \times 8$.
}

TABLE I

BINARIZATION FOR THE PALETTE RUN VALUE.

\begin{tabular}{|l|l|l|}
\hline value & prefix & suffix \\
\hline 0 & 0 & - \\
\hline 1 & 10 & - \\
\hline $2-3$ & 110 & $\mathrm{X}$ \\
\hline $4-7$ & 1110 & $\mathrm{XX}$ \\
\hline $7-15$ & 11110 & $\mathrm{XXX}$ \\
\hline$\cdots$ & $\cdots$ & $\cdots$ \\
\hline
\end{tabular}

dex values can be inferred, thereby eliminating the need to signal the palette sample modes, indices and runs. Furthermore a COPY_ABOVE_MODE may not occur in the first row (or column for vertical traverse scan) and may not follow COPY_ABOVE_MODE. This is used in inferring COPY_INDEX_MODE under these conditions.

\section{Adaptive colour transform}

Much screen content is captured in the RGB colour space. For an image block in RGB colour space, usually there can be strong correlation among different colour components such that a colour space conversion is useful for removing intercolour component redundancy. However, for screen content, there may exist many image blocks containing different features having very saturated colours, which leads to less correlation among colour components. For those blocks, coding directly in the RGB colour space may be more effective. To handle different characteristics of image blocks in screen content, a RGB-to- $Y C_{o} C_{g}$ conversion [36] as shown in the following equation was investigated, and it turned out to be effective.

$$
\left[\begin{array}{l}
Y \\
C_{o} \\
C_{g}
\end{array}\right]=\left[\begin{array}{ccc}
1 / 4 & 1 / 2 & 1 / 4 \\
1 / 2 & 0 & -1 / 2 \\
-1 / 4 & 1 / 2 & -1 / 4
\end{array}\right]\left[\begin{array}{l}
R \\
G \\
B
\end{array}\right]
$$

When this colour transform is used, both the input image block and its corresponding prediction use the same conversion. Because the conversion is linear, it is identical to having the transform applied to residuals in the spatial domain when the prediction processes in different colour components are consistent. Thus, in HEVC-SCC, the conversion is applied 
horizontal traverse scan

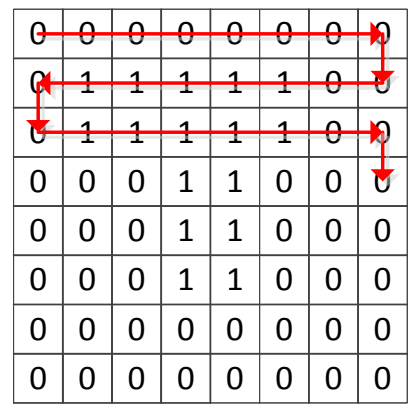

vertical traverse scan

\begin{tabular}{|c|c|c|c|c|c|c|c|}
\hline$\varphi$ & \multicolumn{2}{|c|}{$9 \rightarrow p$} & 0 & 0 & 0 & 0 & 0 \\
\hline$\phi$ & 1 & 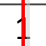 & 1 & 1 & 1 & 0 & 0 \\
\hline$\phi$ & 1 & 7 & 1 & 1 & 1 & 0 & 0 \\
\hline$\phi$ & $\phi$ & $\phi$ & 1 & 1 & 0 & 0 & 0 \\
\hline$\phi$ & $\phi$ & $\phi$ & 1 & 1 & 0 & 0 & 0 \\
\hline$\phi$ & $\phi$ & $\phi$ & 1 & 1 & 0 & 0 & 0 \\
\hline$\phi$ & $\phi$ & $\phi$ & 0 & 0 & 0 & 0 & 0 \\
\hline$t$ & $-\infty$ & 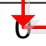 & $\rightarrow 0$ & 0 & 0 & 0 & 0 \\
\hline
\end{tabular}

Fig. 5. Horizontal and vertical traverse scans for the coding of palette indices.

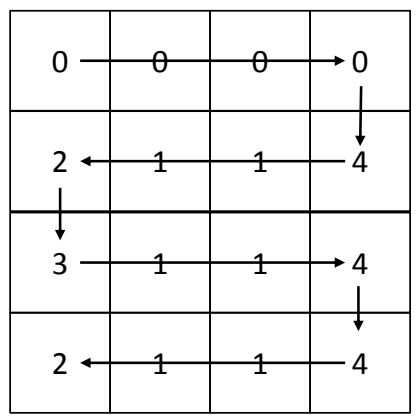

$\begin{array}{ccc}\text { Mode } & \text { Index } & \text { Run } \\ \text { COPY_INDEX } & 0 & 3 \\ \text { COPY_INDEX } & 4 & 0 \\ \text { COPY_INDEX } & 1 & 1 \\ \text { COPY_INDEX } & 2 & 0 \\ \text { COPY_INDEX } & 3 & 0 \\ \text { COPY_ABOVE } & - & 5 \\ \text { COPY_INDEX } & 2 & 0\end{array}$

Fig. 6. Example of palette sample modes, indices, and run values for a $4 \times 4$ block.

on the residual [37], which makes the prediction process for different colour components independent. It is also noted for intra-coded blocks, when the intra prediction directions for different colour components are not the same, the colour transform is not allowed to be used. This limitation is specified because when the intra prediction directions are different, the correlation among colocated samples across colour components is decreased, making the colour transform less effective. The colour transform also changes the norm of different components. To normalize the errors in different colour spaces, when the above transform is used for an image block, a set of QP offsets $(-5,-5,-3)$ is applied to those three colour components [38] during quantization. After the quantization and reconstruction, an inverse transform is applied to the quantized residual so that the reconstruction is still kept in the input colour space.

To limit the dynamic range expansion brought by the colour transform, a lifting-based approximation is used in HEVCSCC. The corresponding colour space is called $Y C_{o} C_{g}-R$. The forward and inverse transform from RGB to $Y C_{o} C_{g}-R$, also from [36], are

$$
\begin{array}{r}
C_{o}=R-B \\
t=B+\left\lfloor C_{o} / 2\right\rfloor \\
C_{g}=G-t \\
Y=t+\left\lfloor C_{g} / 2\right\rfloor
\end{array}
$$

$$
\begin{array}{r}
t=Y-\left\lfloor C_{g} / 2\right\rfloor \\
G=C_{g}+t \\
B=t-\left\lfloor C_{o} / 2\right\rfloor \\
R=B+C_{o}
\end{array}
$$

where $\lfloor x\rfloor$ denotes the greatest integer less than or equal to $x$.

With the forward transform (2), the bit-depth of $Y$ remains the same as that of the input, while for $C_{o}$ and $C_{g}$ components, the bit-depth will be increased by 1 . For lossless coding, (2) and (3) are directly used. While for lossy coding, to keep the bit-depth identical to the residual of the original colour space, an additional right shift is applied to $C_{o}$ and $C_{g}$ components after the forward transform. Correspondingly, an additional left shift is applied before the inverse transform [39].

Even after the adaptive colour transform is applied in the encoder, or in cases when the input video has already undergone a colour transform, there can still be inter-component redundancy that may benefit from the application of crosscomponent prediction, e.g. as shown in [40], [41]. Therefore, CCP may also be applied to CUs which have undergone the adaptive colour transform. It is shown in [42] that both ACT and CCP can significantly improve the coding efficiency for videos in RGB colour space, while ACT might improve more. In [43], Lai et. al. compare performance with different adaptive strategies, which indicates that coding in $\mathrm{YCoCg}$ colour space remarkably improve the coding efficiency, while adaptively choose a better colour space can further improve the performance, especially for RGB videos and lossless coding cases. 
TABLE II

TYPES OF CANDIDATE BVS AND ENCODER RESTRICTIONS ON THEIR USE

\begin{tabular}{ccc}
\hline candidate BVs & $\begin{array}{c}\text { PU size } \\
\text { restriction }\end{array}$ & other restrictions \\
\hline local search region & - & $\begin{array}{c}\text { current CTU and one or more } \\
\text { CTUs to the left (configurable) }\end{array}$ \\
$\begin{array}{c}\text { extended search } \\
\text { region }\end{array}$ & up to $16 \times 16$ & enable or disable (configurable) \\
$\begin{array}{c}\text { Previously used BVs } \\
\text { with same PU size }\end{array}$ & up to $16 \times 16$ & up to $64 \mathrm{BVs}$ \\
\hline
\end{tabular}

\section{Adaptive motion vector resolution}

For camera-captured video, the movement of a real-world object is not necessarily exactly aligned to the sample positions in a camera's sensor. Motion compensation is therefore not limited to using integer sample positions, i.e. fractional motion compensation is used to improve compression efficiency. Computer-generated screen content video, however, is often generated with knowledge of the sample positions, resulting in motion that is discrete or precisely aligned with sample positions in the picture. For this kind of video, integer motion vectors may be sufficient for representing the motion. Savings in bit-rate can be achieved by not signalling the fractional portion of the motion vectors.

In HEVC-SCC, adaptive motion vector resolution (AMVR) [44] defines a slice-level flag to indicate that the current slice uses integer (full-pel) motion vectors for luma samples. If the flag is true, then the motion vector predictions, motion vector differences, and resulting motion vectors assume only integer values, so the bits representing the fractional values do not need to be signalled. To minimize differences between HEVC-SCC and HEVC version 1, the decoded integer motion vectors are simply left-shifted by one or two bits, so the rest of the motion vector processing is unchanged as the fractional bits are zero.

\section{ENCODING ALGORITHMS}

Because of the introduction of new coding tools as described in Section III and different characteristics of screen content, new encoding algorithms were introduced during the development of HEVC-SCC. These methods are part of the HEVC-SCC test model 3 [45]. It should be understood that these methods are not normative but help improve the coding efficiency of the HEVC-SCC coding tools. The new algorithms that are much different from conventional video encoding methods are described in this session for a better understanding of HEVC-SCC.

\section{A. Intra block vector search}

In order to decide whether to use IBC mode for a CU, a rate-distortion $(\mathrm{RD})$ cost is calculated for the $\mathrm{CU}$. Block matching (BM) is performed at the encoder to find the optimal BV for each prediction unit. Depending on the size of the prediction unit (PU), the following three types of candidate $\mathrm{BVs}$ are evaluated as shown in Table II
The RD cost for each candidate $\mathrm{BV}$ is evaluated as:

$$
\mathrm{RD}_{-} \text {cost }_{\mathrm{Y}}=\mathrm{SAD}_{\text {luma }}+\lambda \times \mathrm{BV}_{\text {bits }}
$$

where SAD is the sum of absolute differences between samples in a block and corresponding samples in the matching block, and $\mathrm{BV}_{\text {bits }}$ is the number of bits needed to signal the BV. Note that only the SAD for the luma block is used at this stage. Once the RD cost for all the candidate vectors is evaluated, the four candidate $\mathrm{BVs}$ with the least $\mathrm{RD}$ cost are chosen. The candidate with the best RD cost is chosen as the optimal BV. In this case, the RD cost is evaluated based on both luma and chroma as follows:

$$
\mathrm{RD} \__{\text {cost }}{ }_{\mathrm{YUV}}=\mathrm{SAD}_{\text {luma }}+\mathrm{SAD}_{\text {chroma }}+\lambda \times \mathrm{BV}_{\text {bits }}
$$

For $8 \times 8$ PUs, the entire picture region conforming to the restrictions on the predictor block as described in Section III-A is searched for the optimal BV using a hash-based search. Each node in the hash table records the position of each BV candidate in the picture. Only the block vector candidates that have the same hash entry value as that of the current block are examined. For PU sizes up to $16 \times 16$, with the exception of $8 \times 8$, an extended area may be searched for the optimal $\mathrm{BV}$. In this case, block vectors in the region conforming to the restrictions on the predictor block as described in Section III-A with at least one zero component (horizontal or vertical) are considered as candidate BVs.

The 16-bit hash entries for the current block and the reference block are calculated using the original sample values. Let $\operatorname{grad}_{\mathrm{BLK}}$ denote the gradient of the $8 \times 8$ block and let $d c 0, d c 1, d c 2$, and $d c 3$ denote the DC values of the four $4 \times 4$ sub-blocks of the $8 \times 8$ block. Then, the 16-bit hash entry $H$ is calculated as:

$$
\begin{aligned}
H= & \operatorname{msb}(d c 0,3) \ll 13+\operatorname{msb}(d c 1,3) \ll 10+ \\
& \operatorname{msb}(d c 2,3) \ll 7+\operatorname{msb}(d c 3,3) \ll 4+ \\
& \operatorname{msb}\left(\operatorname{grad}_{\mathrm{BLK}}, 4\right)
\end{aligned}
$$

where $\operatorname{msb}(X, n)$ represents the $n$ most significant bits of $X$.

The gradient is calculated as follows: First for each sample in the block except for the first line and first column, gradX is calculated as the absolute difference between the current sample and the sample to the left. Similarly $\operatorname{grad} Y$ is calculated as the absolute difference between the current sample and the sample above. Then grad for the sample is set equal to average of $\operatorname{gradX}$ and $\operatorname{grad} Y$. The final $\operatorname{grad}_{\mathrm{BLK}}$ for the block is the cumulative sum of the per-sample grad values over the block.

\section{B. Inter search}

Large motion is often observed in screen content, which makes the conventional motion search computationally prohibitive. However, because digitally-captured screen content is usually noiseless, it is possible to use fast search methods in the encoder to identify the corresponding block in a reference picture. In the reference software, a hash-based block matching algorithm [46] is used to search for identical blocks in reference pictures. For a reference picture, a 16-bit cyclic 
redundancy check (CRC) value is calculated using the original sample values for a block starting from any sample position and having sizes of $8 \times 8,16 \times 16,32 \times 32$ and $64 \times 64$. An 18-bit hash value is formed comprising the 16-bit CRC and 2 bits representing the block size. An inverted index is used to index every block having the same hash value. For a $\mathrm{CU}$ with a $2 \mathrm{~N} \times 2 \mathrm{~N}$ prediction unit, the hash value is calculated, and with this hash value an exact match can be searched for using the inverted index in linear time, regardless of the search range and number of reference pictures. To reduce the memory requirement for storing the hash table, a pre-filtering process is applied to exclude blocks that can be easily predicted using intra prediction. For those blocks, it is not necessary to perform block matching. When a block cannot find an exact match in the reference picture, as is the case for most camera-captured material, conventional motion estimation is used. For simple screen content material, most blocks can have exact matches. Thus, the motion estimation search process is often be skipped, which can significantly reduce the encoding complexity.

\section{Palette mode encoding}

To evaluate the RD cost of encoding a $\mathrm{CU}$ in palette mode a palette table for the $\mathrm{CU}$ is derived as follows. A modified k-means clustering method is used to derive a palette table in the lossy case. The palette table is initialized to have no entries. Then, for each sample in the $\mathrm{CU}$, the nearest palette entry (in terms of SAD) is determined. If the SAD is within a threshold value, the sample is added to the cluster corresponding to the nearest palette entry. Otherwise a new palette entry is created to be equal to the sample value. After processing all the samples in the $\mathrm{CU}$, each palette entry is updated by the centroid of the cluster. Then, the palette entries are sorted based on the number of entries in their associated clusters. There is a limit on the maximum palette size, which is signalled in the sequence parameter set. If the number of palette entries exceeds this limit, the least frequent clusters are eliminated and the sample values belonging to those clusters are converted to escape samples.

As discussed in Section III-B, if a palette entry is already in the palette predictor, the cost of including it in the current palette is small. On the other hand, when the centroid is not in the palette predictor, all the components have to be signalled explicitly in the bit-stream, resulting in much higher cost. Hence a rate distortion analysis is performed to determine whether assigning a cluster to an existing palette predictor entry has a lower RD cost. If this is the case, then the centroid is replaced by the palette predictor entry. After this step, duplicate palette entries are removed. Also, if a cluster includes a single entry that is not contained in the palette predictor, the entry is converted to an escape sample. For lossless coding a slightly different method of palette derivation based on histogram of the CU samples is used.

Once the palette table and assignment of CU sample values to palette indices are determined, a rate-based encoder optimization is performed to determine the palette_run_type_flag and run values [47]. For example, assuming that index mode is chosen for the sample, a run value is determined. Then, the per-sample cost in bits for coding the index and run value is calculated. This cost is compared to the per-sample bit cost for coding the run value assuming COPY_ABOVE_MODE is chosen. The run type with the lower per-sample bit cost is chosen. The decision is greedy in the sense that sometimes when COPY_ABOVE_MODE is chosen, the cost of coding an index is just postponed to a future run. More sophisticated strategies are possible. For example, instead of first performing index assignment and then making the decision about palette_run_type_flag and run values, it may be possible to make these decisions jointly. As a simple example, by forcing a sample value to map to a different index, it may be possible to extend a run, thereby lowering the RD cost. Such strategies are currently not considered in HEVC-SCC test model 3 encoder.

\section{Decision for adaptive colour transform}

When the adaptive colour transform is enabled, a coding unit can choose to perform the colour transform described in III-C on the residual of three colour components. In the current test model, the encoder simply compares the R-D costs of coding in both modes (with or without the colour transform) and selects one with the least cost. Thus, the encoding time increases accordingly.

\section{E. Decision for adaptive motion vector resolution}

For each slice, an encoder can choose to use full-pel or fractional motion vectors for luma samples. In the current test model, instead of checking both options and comparing the $\mathrm{RD}$ costs, a fast algorithm is used to make the decision for the entire slice. The basic idea of this algorithm is to determine whether most blocks in the current slice have exact matching blocks with full-pel displacements in the reference picture.

Similar to the inter search scheme described earlier, hash values are precomputed for every possible $8 \times 8$ block in the reference picture. Next, the current slice is divided into nonoverlapped $8 \times 8$ blocks. For each block, the corresponding hash value is calculated, and an exact match for each current block's hash is searched for in the list of reference block hashes, without considering the case of hash collision. A match indicates that an integer motion vector would yield an exact match, without the need to actually perform the motion search. If exact matches are found for most of the $8 \times 8$ blocks, then full-pel motion vectors will be used for the slice. For the detailed algorithms, readers can refer to [44].

\section{Performance analysis}

Simulations were conducted to evaluate the new coding tools in HEVC-SCC and compare the coding efficiency of HEVC-SCC with HEVC-RExt and H.264/AVC. For H.264/AVC, HEVC-RExt and HEVC-SCC, the reference software JM-18.6, HM-16.4 and SCM-3.0/SCM-4.0 were used, respectively. 


\section{A. Test conditions}

The common test conditions (CTC) used during the development of HEVC-SCC [48] were used to generate the results presented in this section. These test conditions include video sequences comprising a variety of resolutions and content types. Table III lists the 4:4:4 sequences. In the category column, "TGM", "CC", "M" and "A" stand for text and graphics with motion, camera-captured content, mixed content (i.e. content having both TGM and CC) and animation. For each sequence, two colour formats are tested. One is in RGB and the other is in $\mathrm{YCbCr}$, denoted as $\mathrm{YUV}$ in the tables). Table IV lists the 4:2:0 sequences. All of them are in $\mathrm{YCbCr}$ colour format. As indicated by their names, several of the 4:2:0 sequences are generated from their 4:4:4 correspondences.

TABLE III

TEST SEQUenCES (4:4:4) IN THE CTC.

\begin{tabular}{rrc}
\hline Resolution & Sequence name & Category \\
\hline & sc_flyingGraphics_1920x1080_60_8bit & TGM \\
sc_desktop_1920x1080_60_8bit & TGM \\
1920x1080 & sc_console_1920x1080_60_8bit & TGM \\
& MissionControlClip3_1920x1080_60p_8b444 & M \\
& EBURainFruits_1920x1080_50_10bit & CC \\
& Kimono1_1920x1080_24_10bit & CC \\
\hline \multirow{2}{*}{$1280 \times 720$} & sc_web_browsing_1280x720_30_8bit & TGM \\
& sc_map_1280x720_60_8bit & TGM \\
& sc_programming_1280x720_60_8bit & TGM \\
\multirow{2}{*}{$2560 x 1440$} & sc_SlideShow_1280x720_20_8bit & TGM \\
& sc_robot_1280x720_30_8bit & A \\
\hline \multirow{2}{*}{ Basketball_Screen_2560x1440_60p_8b444 } & M \\
& MissionControlClip2_2560x1440_60p_8444 & M \\
\hline
\end{tabular}

TABLE IV

TEST SEQuenCES (4:2:0) IN THE CTC.

\begin{tabular}{crc}
\hline Resolution & Sequence name & Category \\
\hline \multirow{3}{*}{$1920 \times 1080$} & sc_flyingGraphics_1920x1080_60_8bit_420 & TGM \\
& sc_desktop_1920x1080_60_8bit_420 & TGM \\
& sc_console_1920x1080_60_8bit_420 & TGM \\
& MissionControlClip3_1920x1080_60p_8b420 & M \\
\hline & sc_web_browsing_1280x720_30_8bit_420_r1 & TGM \\
1280x720 & sc_map_1280x720_60_8bit_420 & TGM \\
& sc_programming_1280x720_60_8bit_420 & TGM \\
& sc_SlideShow_1280x720_20_8bit_420 & TGM \\
\hline \multirow{2}{*}{$2560 \times 1440$} & sc_robot_1280x720_30_8bit_420 & A \\
\hline \multirow{2}{*}{$1024 \times 768$} & Basketball_Screen_2560x1440_60p_8b420 & M \\
& MissionControlClip2_2560x1440_60p_8420 & M \\
\hline
\end{tabular}

Three test configurations, i.e. all intra (AI), random access (RA) and low-delay B (LB) were used. For lossy coding, four QPs, $\{22,27,32,37\}$, were applied. More details can be found in [48].

\section{B. Performance analysis of coding tools}

To verify the effectiveness of coding tools described in Section III, Tables V, VI, VII and VIII show the coding performance compared to an anchor not having the specific coding tool in SCM-3.0, for 4:4:4 sequences ${ }^{2}$. Only the BDrate measures of the first colour component are listed due to space limitations. From these tables, it can be seen that both IBC and palette modes are very effective for videos in the TGM and M categories. For camera-captured content, both tools neither help nor harm the coding efficiency noticeably. Another observation is that the coding gain for AI is larger than that for RA or LB, because both IBC and palette modes are for intra coding.

From Table VII, it can be observed that ACT improves the coding of RGB videos significantly, regardless of categories. In contrast, the performance gains of ACT are limited when the video is already in the YUV colour format.

TABLE V

COMPARISON OF SCM-3.0 WITH VERSUS WITHOUT IBC (BD-RATE CHANGE).

\begin{tabular}{crccc}
\hline & & AI & RA & LB \\
\hline \multirow{4}{*}{ RGB } & TGM & $-31.3 \%$ & $-19.1 \%$ & $-10.6 \%$ \\
& M & $-28.9 \%$ & $-19.2 \%$ & $-8.9 \%$ \\
& A & $-1.1 \%$ & $-0.5 \%$ & $-0.1 \%$ \\
& CC & $-0.1 \%$ & $0.0 \%$ & $0.0 \%$ \\
\hline \multirow{4}{*}{ YUV } & TGM & $-32.5 \%$ & $-19.1 \%$ & $-9.8 \%$ \\
& M & $-29.5 \%$ & $-19.9 \%$ & $-8.9 \%$ \\
& A & $-1.6 \%$ & $-0.5 \%$ & $0.1 \%$ \\
& CC & $-0.2 \%$ & $0.0 \%$ & $0.1 \%$ \\
\hline
\end{tabular}

TABLE VI

COMPARISON OF SCM-3.0 WITH VERSUS WITHOUT PALETTE (BD-RATE CHANGE).

\begin{tabular}{crccc}
\hline & & AI & RA & LB \\
\hline \multirow{4}{*}{ RGB } & TGM & $-15.5 \%$ & $-10.5 \%$ & $-6.8 \%$ \\
& M & $-3.7 \%$ & $-2.6 \%$ & $-1.5 \%$ \\
& A & $0.0 \%$ & $-0.1 \%$ & $0.0 \%$ \\
& CC & $0.0 \%$ & $0.0 \%$ & $0.0 \%$ \\
\hline \multirow{4}{*}{ YUV } & TGM & $-16.2 \%$ & $-11.1 \%$ & $-6.8 \%$ \\
& M & $-5.9 \%$ & $-4.4 \%$ & $-2.7 \%$ \\
& A & $0.1 \%$ & $0.2 \%$ & $0.1 \%$ \\
& CC & $0.0 \%$ & $0.1 \%$ & $0.1 \%$ \\
\hline
\end{tabular}

TABLE VII

COMPARISON OF SCM-3.0 WITH VERSUS WITHOUT ACT (BD-RATE CHANGE).

\begin{tabular}{crccc}
\hline & & AI & RA & LB \\
\hline \multirow{4}{*}{ RGB } & TGM & $-9.6 \%$ & $-11.6 \%$ & $-11.1 \%$ \\
& M & $-16.6 \%$ & $-23.1 \%$ & $-23.7 \%$ \\
& A & $-24.5 \%$ & $-24.9 \%$ & $-24.0 \%$ \\
& CC & $-24.5 \%$ & $-27.5 \%$ & $-26.1 \%$ \\
\hline \multirow{4}{*}{ YUV } & TGM & $-0.4 \%$ & $-0.7 \%$ & $-1.0 \%$ \\
& M & $0.1 \%$ & $0.4 \%$ & $0.4 \%$ \\
& A & $0.1 \%$ & $-0.1 \%$ & $0.0 \%$ \\
& CC & $0.1 \%$ & $0.5 \%$ & $0.3 \%$ \\
\hline
\end{tabular}

\footnotetext{
${ }^{2}$ For individual tool comparisons, SCM-3.0 is used because it already contains all coding tools mentioned above. The improvements of later versions of SCM are mainly for 4:2:0 sequences.
} 
TABLE VIII

COMPARISON OF SCM-3.0 WITH VERSUS WITHOUT AMVR (BD-RATE CHANGE).

\begin{tabular}{crcc}
\hline & & RA & LB \\
\hline & TGM & $-1.4 \%$ & $-2.2 \%$ \\
RGB & M & $0.0 \%$ & $0.0 \%$ \\
& A & $0.0 \%$ & $0.0 \%$ \\
& CC & $0.0 \%$ & $0.0 \%$ \\
\hline & TGM & $-1.5 \%$ & $-2.4 \%$ \\
YUV & M & $0.0 \%$ & $-0.1 \%$ \\
& A & $0.0 \%$ & $0.0 \%$ \\
& CC & $0.0 \%$ & $0.0 \%$ \\
\hline
\end{tabular}

\section{Performance comparison with existing standards}

Tables IX and XI show the performance comparison between SCM-4.0 and HM-16.4, for lossy and lossless coding respectively. For lossy coding, the BD-rate changes for all three colour components are shown. For lossless coding, the average bit-saving percentages are listed. Significant improvements in compression efficiency are achieved, especially for videos in the TGM and $\mathrm{M}$ categories. Decreases in BD-rate of more than $50 \%$ indicate that SCM-4.0 can perform with more than twice the compression efficiency of HM-16.4. For comparisons with H.264/AVC, Table XIII and XV show the coding performance comparison between SCM-4.0 and JM18.6 .

TABLE XI

LOSSLESS CODING PERFORMANCE COMPARISON BETWEEN SCM-4.0 AND HM-16.4 FOR 4:4:4 SEQUENCES (BD-RATE CHANGE).

\begin{tabular}{crccc}
\hline Colour format & Category & AI & RA & LB \\
\hline \multirow{4}{*}{ RGB } & TGM & $-45.8 \%$ & $-35.2 \%$ & $-32.2 \%$ \\
& M & $-24.3 \%$ & $-6.3 \%$ & $-3.9 \%$ \\
& $\mathrm{~A}$ & $-4.4 \%$ & $-1.1 \%$ & $-1.1 \%$ \\
& $\mathrm{CC}$ & $-0.2 \%$ & $0.4 \%$ & $0.4 \%$ \\
\hline \multirow{4}{*}{ YUV } & TGM & $-46.7 \%$ & $-36.4 \%$ & $-33.3 \%$ \\
& $\mathrm{M}$ & $-23.9 \%$ & $-6.3 \%$ & $-3.8 \%$ \\
& $\mathrm{~A}$ & $-1.7 \%$ & $-0.3 \%$ & $-0.3 \%$ \\
& $\mathrm{CC}$ & $0.0 \%$ & $0.0 \%$ & $0.0 \%$ \\
\hline
\end{tabular}

TABLE XII

LOSSLESS CODING PERFORMANCE COMPARISON BETWEEN SCM-4.0 AND HM-16.4 FOR 4:2:0 SEQUENCES (BD-RATE CHANGE).

\begin{tabular}{rccc}
\hline Category & AI & RA & LB \\
\hline TGM & $-34.1 \%$ & $-23.9 \%$ & $-21.1 \%$ \\
M & $-21.6 \%$ & $-6.0 \%$ & $-3.5 \%$ \\
A & $-0.7 \%$ & $-0.2 \%$ & $-0.1 \%$ \\
\hline
\end{tabular}

\section{Vi. CONCLusions}

The HEVC screen content coding extension was developed to leverage the unique characteristics of computer-generated and digitally-captured videos. The intra block copy mode takes advantage of the presence of exact copies of blocks between different pictures in a video sequence. Palette mode targets blocks having a limited number of unique colour values, which frequently occur in computer-generated pictures. The adaptive
TABLE XV

LOSSLESS CODING PERFORMANCE COMPARISON BETWEEN SCM-4.0 AND JM-18.6 FOR 4:4:4 SEQUENCES (BD-RATE CHANGE).

\begin{tabular}{crccc}
\hline Colour format & Category & AI & RA & LB \\
\hline \multirow{4}{*}{ RGB } & TGM & $-66.7 \%$ & $-59.1 \%$ & $-58.9 \%$ \\
& M & $-44.4 \%$ & $-28.4 \%$ & $-26.6 \%$ \\
& A & $-20.9 \%$ & $-14.3 \%$ & $-13.0 \%$ \\
& CC & $-6.9 \%$ & $-2.9 \%$ & $-2.8 \%$ \\
\hline \multirow{3}{*}{ YUV } & TGM & $-53.5 \%$ & $-47.7 \%$ & $-47.4 \%$ \\
& M & $-29.3 \%$ & $-16.7 \%$ & $-14.9 \%$ \\
& A & $-5.0 \%$ & $-7.6 \%$ & $-6.2 \%$ \\
& CC & $-0.7 \%$ & $-1.4 \%$ & $-1.4 \%$ \\
\hline
\end{tabular}

TABLE XVI

LOSSLESS CODING PERFORMANCE COMPARISON BETWEEN SCM-4.0 AND JM-18.6 FOR 4:2:0 SEQUENCES (BD-RATE CHANGE).

\begin{tabular}{rccc}
\hline Category & AI & RA & LB \\
\hline TGM & $-44.2 \%$ & $-39.8 \%$ & $-36.6 \%$ \\
M & $-26.9 \%$ & $-16.9 \%$ & $-14.1 \%$ \\
A & $-4.5 \%$ & $-9.6 \%$ & $-7.7 \%$ \\
\hline
\end{tabular}

colour transform performs an in-loop colour space conversion from RGB to $\mathrm{YCoCg}$, and an adaptive motion vector resolution eliminates the need to signal fractional motion vectors for computer-generated video. With these new tools, HEVC-SCC is capable of performing with more than twice the compression efficiency of HEVC-RExt for material having primarily text and graphics or mixed content.

\section{ACKNOWLEDGMENT}

The authors would like to thank the many experts of the aforementioned standardization organizations whose joint efforts and contributions led to the creation of the screen content coding extension.

\section{REFERENCES}

[1] G. J. Sullivan, J. Ohm, W.-J. Han, and T. Wiegand, "Overview of the High Efficiency Video Coding (HEVC) standard," IEEE Trans. Circuits Syst. Video Technol., vol. 22, no. 12, pp. 1649-1668, 2012.

[2] ITU-T and ISO/IEC JTC1, Advanced Video Coding for Generic AudioVisual Services. ITU-T Rec. H.264 and ISO/IEC 14496-10 (AVC), May. 2003 (and subsequent editions).

[3] H. Yu, K. McCann, R. Cohen, and P. Amon, "Requirements for an extension of HEVC for coding of screen content," ISO/IEC JTC 1/SC 29/WG 11 Requirements subgroup, San Jose, California, USA, document MPEG2014/N14174, Jan. 2014.

[4] C. Lan, J. Xu, F. Wu, and G. J. Sullivan, "Screen content coding," 2nd JCT-VC meeting, Geneva, Switzerland, document JCTVC-B084, Jul. 2010.

[5] W. Gao, G. Cook, M. Yang, J. Song, and H. Yu, "Near lossless coding for screen content," 6th JCT-VC meeting, Torino, Italy, document JCTVCF564, Jul. 2011

[6] C. Lan, X. Peng, J. Xu, and F. Wu, "Intra and inter coding tools for screen contents," 5th JCT-VC meeting, Geneva, Switzerland, document JCTVC-E145, Mar. 2011.

[7] S. Wang and T. Lin, "4:4:4 screen content coding using macroblockadaptive mixed chroma-sampling-rate," 8th JCT-VC meeting, San Jose, California, USA, document JCTVC-H0073, Feb. 2012.

[8] T. Lin, P. Zhang, S. Wang, and K. Zhou, "4:4:4 screen content coding using dual-coder mixed chroma-sampling-rate (DMC) techniques," 9th JCT-VC meeting, Geneva, Switzerland, document JCTVC-I0272, Apr. 2012. 
TABLE IX

LOSSY CODING PERFORMANCE COMPARISON BETWEEN SCM-4.0 AND HM-16.4 FOR 4:4:4 SEQUENCES (BD-RATE CHANGE).

\begin{tabular}{crccccccccc}
\hline \multirow{2}{*}{ Colour format } & & \multicolumn{3}{c}{ AI } & & \multicolumn{3}{c}{ RA } & \multicolumn{3}{c}{ LB } \\
& Category & $\mathrm{G} / \mathrm{Y}$ & $\mathrm{B} / \mathrm{U}$ & $\mathrm{R} / \mathrm{V}$ & $\mathrm{G} / \mathrm{Y}$ & $\mathrm{B} / \mathrm{U}$ & $\mathrm{R} / \mathrm{V}$ & $\mathrm{G} / \mathrm{Y}$ & $\mathrm{B} / \mathrm{U}$ & $\mathrm{R} / \mathrm{V}$ \\
\hline \multirow{3}{*}{ RGB } & $\mathrm{TGM}$ & $-64.5 \%$ & $-60.9 \%$ & $-62.0 \%$ & $-56.9 \%$ & $-51.0 \%$ & $-53.1 \%$ & $-50.8 \%$ & $-42.9 \%$ & $-45.3 \%$ \\
& $\mathrm{M}$ & $-54.8 \%$ & $-49.7 \%$ & $-49.5 \%$ & $-50.2 \%$ & $-42.4 \%$ & $-42.0 \%$ & $-41.7 \%$ & $-29.2 \%$ & $-28.2 \%$ \\
& $\mathrm{~A}$ & $-26.3 \%$ & $-19.5 \%$ & $-16.8 \%$ & $-26.2 \%$ & $-17.3 \%$ & $-12.9 \%$ & $-24.4 \%$ & $-11.9 \%$ & $-5.5 \%$ \\
& $\mathrm{CC}$ & $-25.6 \%$ & $-5.5 \%$ & $-10.4 \%$ & $-28.3 \%$ & $-5.8 \%$ & $-14.4 \%$ & $-26.1 \%$ & $-1.6 \%$ & $-11.9 \%$ \\
\hline \multirow{5}{*}{$\mathrm{YUV}$} & $\mathrm{TGM}$ & $-57.4 \%$ & $-61.3 \%$ & $-62.8 \%$ & $-48.0 \%$ & $-52.6 \%$ & $-55.3 \%$ & $-40.5 \%$ & $-44.9 \%$ & $-47.4 \%$ \\
& $\mathrm{M}$ & $-45.2 \%$ & $-50.9 \%$ & $-50.8 \%$ & $-36.7 \%$ & $-45.1 \%$ & $-44.8 \%$ & $-23.8 \%$ & $-33.6 \%$ & $-33.3 \%$ \\
& $\mathrm{~A}$ & $-1.2 \%$ & $-10.9 \%$ & $-7.6 \%$ & $-0.4 \%$ & $-10.1 \%$ & $-6.8 \%$ & $0.0 \%$ & $-7.0 \%$ & $-4.9 \%$ \\
& $\mathrm{CC}$ & $0.4 \%$ & $0.0 \%$ & $-0.2 \%$ & $0.6 \%$ & $-0.2 \%$ & $-0.3 \%$ & $0.6 \%$ & $-0.3 \%$ & $-0.2 \%$ \\
\hline
\end{tabular}

TABLE X

LOSSY CODING PERFORMANCE COMPARISON BETWEEN SCM-4.0 AND HM-16.4 FOR 4:2:0 SEQUENCES (BD-RATE CHANGE).

\begin{tabular}{|c|c|c|c|c|c|c|c|c|c|}
\hline \multirow[b]{2}{*}{ Category } & \multicolumn{3}{|c|}{ AI } & \multicolumn{3}{|c|}{$\mathbf{R A}$} & \multicolumn{3}{|c|}{ LB } \\
\hline & $\mathrm{G} / \mathrm{Y}$ & $\mathrm{B} / \mathrm{U}$ & $\mathrm{R} / \mathrm{V}$ & $\mathrm{G} / \mathrm{Y}$ & $\mathrm{B} / \mathrm{U}$ & $\mathrm{R} / \mathrm{V}$ & G/Y & $\mathrm{B} / \mathrm{U}$ & $\mathrm{R} / \mathrm{V}$ \\
\hline TGM & $-49.0 \%$ & $-49.3 \%$ & $-50.5 \%$ & $-39.4 \%$ & $-40.6 \%$ & $-42.2 \%$ & $-32.7 \%$ & $-33.2 \%$ & $-34.4 \%$ \\
\hline M & $-36.6 \%$ & $-37.6 \%$ & $-37.6 \%$ & $-29.4 \%$ & $-31.2 \%$ & $-31.5 \%$ & $-18.0 \%$ & $-18.7 \%$ & $-19.5 \%$ \\
\hline A & $-7.3 \%$ & $-11.7 \%$ & $-10.7 \%$ & $-3.8 \%$ & $-12.4 \%$ & $-9.9 \%$ & $-2.0 \%$ & $-8.2 \%$ & $-5.7 \%$ \\
\hline
\end{tabular}

TABLE XIII

LOSSY CODING PERFORMANCE COMPARISON BETWEEN SCM-4.0 AND JM-18.6 FOR 4:4:4 SEQUENCES (BD-RATE CHANGE).

\begin{tabular}{crrcccccccc}
\hline \multirow{3}{*}{ Colour format } & & \multicolumn{3}{c}{ AI } & & \multicolumn{3}{c}{ RA } & \multicolumn{3}{c}{ LB } \\
& Category & G/Y & B/U & R/V & G/Y & B/U & R/V & G/Y & B/U & R/V \\
\hline \multirow{3}{*}{ RGB } & TGM & $-86.1 \%$ & $-83.5 \%$ & $-84.1 \%$ & $-80.4 \%$ & $-76.1 \%$ & $-77.4 \%$ & $-77.7 \%$ & $-73.0 \%$ & $-74.4 \%$ \\
& M & $-80.1 \%$ & $-76.2 \%$ & $-76.0 \%$ & $-74.3 \%$ & $-68.2 \%$ & $-67.3 \%$ & $-69.7 \%$ & $-60.8 \%$ & $-59.8 \%$ \\
& A & $-52.4 \%$ & $-45.0 \%$ & $-40.1 \%$ & $-54.8 \%$ & $-49.5 \%$ & $-43.2 \%$ & $-56.4 \%$ & $-51.0 \%$ & $-43.1 \%$ \\
& CC & $-58.4 \%$ & $-35.6 \%$ & $-44.3 \%$ & $-63.3 \%$ & $-42.6 \%$ & $-51.5 \%$ & $-60.1 \%$ & $-36.5 \%$ & $-48.2 \%$ \\
\hline \multirow{5}{*}{ YUV } & TGM & $-74.6 \%$ & $-75.0 \%$ & $-77.0 \%$ & $-68.1 \%$ & $-70.4 \%$ & $-73.3 \%$ & $-65.4 \%$ & $-67.6 \%$ & $-70.5 \%$ \\
& M & $-63.6 \%$ & $-64.8 \%$ & $-64.8 \%$ & $-56.9 \%$ & $-63.2 \%$ & $-63.1 \%$ & $-51.5 \%$ & $-60.5 \%$ & $-60.6 \%$ \\
& A & $-23.4 \%$ & $-35.5 \%$ & $-29.3 \%$ & $-32.2 \%$ & $-48.0 \%$ & $-41.8 \%$ & $-39.0 \%$ & $-59.6 \%$ & $-54.6 \%$ \\
& CC & $-26.5 \%$ & $-18.5 \%$ & $-25.4 \%$ & $-40.0 \%$ & $-42.2 \%$ & $-42.6 \%$ & $-39.8 \%$ & $-51.5 \%$ & $-53.6 \%$ \\
\hline
\end{tabular}

TABLE XIV

LOSSY CODING PERFORMANCE COMPARISON BETWEEN SCM-4.0 AND JM-18.6 FOR 4:2:0 SEQUENCES (BD-RATE CHANGE).

\begin{tabular}{|c|c|c|c|c|c|c|c|c|c|}
\hline \multirow[b]{2}{*}{ Category } & \multicolumn{3}{|c|}{ AI } & \multicolumn{3}{|c|}{ RA } & \multicolumn{3}{|c|}{ LB } \\
\hline & $\mathrm{G} / \mathrm{Y}$ & $\mathrm{B} / \mathrm{U}$ & $\mathrm{R} / \mathrm{V}$ & $\mathrm{G} / \mathrm{Y}$ & $\mathrm{B} / \mathrm{U}$ & $R / V$ & $\mathrm{G} / \mathrm{Y}$ & $\mathrm{B} / \mathrm{U}$ & $\mathrm{R} / \mathrm{V}$ \\
\hline TGM & $-69.9 \%$ & $-64.4 \%$ & $-65.3 \%$ & $-62.3 \%$ & $-61.6 \%$ & $-62.6 \%$ & $-60.3 \%$ & $-58.7 \%$ & $-59.5 \%$ \\
\hline M & $-57.4 \%$ & $-52.2 \%$ & $-53.1 \%$ & $-51.8 \%$ & $-51.7 \%$ & $-52.7 \%$ & $-47.0 \%$ & $-46.0 \%$ & $-47.7 \%$ \\
\hline A & $-31.7 \%$ & $-33.3 \%$ & $-33.5 \%$ & $-36.4 \%$ & $-45.2 \%$ & $-44.2 \%$ & $-39.5 \%$ & $-45.0 \%$ & $-43.9 \%$ \\
\hline
\end{tabular}

[9] ISO/IEC JTC1/SC29/WG11, "Joint call for proposals for coding of screen content," ITU-T Q6/16 Visual Coding and ISO/IEC JTC1/SC29/WG11 Coding of Moving Pictures and Audio, San Jose, California, USA, document MPEG2014/N14175, Jan. 2014.

[10] R. Joshi and J. Xu, "HEVC Screen Content Coding Draft Text 1," 18th JCT-VC meeting, Sapporo, Japan, document JCTVC-R1005, Jul. 2014.

[11] — , "HEVC Screen Content Coding Draft Text 2," 19th JCT-VC meeting, Strasbourg, France, document JCTVC-S1005, Nov. 2014.

[12] J. Boyce, J. Chen, Y. Chen, D. Flynn, M. M. Hannuksela, M. Naccari, C. Rosewarne, K. Sharman, J. Sole, G. J. Sullivan, T. Suzuki, G. Tech, Y.-K. Wang, K. Wegner, and Y. Ye, "Edition 2 Draft Text of High Efficiency Video Coding (HEVC), Including Format Range (RExt), Scalability (SHVC), and Multi-View (MV-HEVC) Extensions," 18th JCT-VC meeting, Sapporo, Japan, document JCTVC-R1013, Jul. 2014.

[13] M. Narroschke, "Extending H.264/AVC by an adaptive coding of the prediction error," in Picture Coding Symposium, PCS2006, Beijing, China, Apr. 2006.

[14] C. Lan, J. Xu, G. J. Sullivan, and F. Wu, "Intra transform skipping," 9th JCT-VC meeting, Geneva, Switzerland, document JCTVC-I0408, Apr. 2012.
[15] X. Peng, C. Lan, J. Xu, and G. J. Sullivan, "Inter transform skipping," 10th JCT-VC meeting, Stockholm, Sweden, document JCTVC-I0408, Jul. 2012.

[16] R. Cohen and A. Vetro, "AHG8: Impact of transform skip on new screen content material," 12th JCT-VC meeting, Geneva, Switzerland, document JCTVC-L0428, Jan. 2013.

[17] X. Peng, J. Xu, L. Guo, J. Sole, and M. Karczewicz, "Non-RCE2: Transform skip on large TUs," 14th JCT-VC meeting, Vienna, Austria, document JCTVC-NO288, Jul. 2013.

[18] J. An, L. Zhao, Y.-W. Huang, and S. Lei, "Residue scan for intra transform skip mode," 10th JCT-VC meeting, Stockholm, Sweden, document JCTVC-J0053, Jun. 2012.

[19] D. He, J. Wang, and G. Martin-Cocher, "Rotation of residual block for transform skipping," 10th JCT-VC meeting, Stockholm, Sweden, document JCTVC-J0093, Jun. 2012.

[20] X. Peng, B. Li, and J. Xu, "On residual rotation for inter and intra bc modes," 15th JCT-VC meeting, Geneva, Switzerland, document JCTVCO0186, Oct. 2013.

[21] S. L. an I.-K. Kim and C. Kim, "AHG7: Residual DPCM for HEVC lossless coding," 12th JCT-VC meeting, Geneva, Switzerland, document 
JCTVC-L0117, Jan. 2013.

[22] R. Joshi, J. Sole, and M. Karczewicz, "AHG8: Residual DPCM for visually lossless coding," 13th JCT-VC meeting, Incheon, Korea, document JCTVC-M0351, Apr. 2013.

[23] M. Naccari, M. Mrak, A. Gabriellini, S. Blasi, and E. Izquierdo, "Interprediction residual DPCM," 13th JCT-VC meeting, Incheon, Korea, document JCTVC-M0442, Apr. 2013.

[24] T. Nguyen, A. Khairat, and D. Marpe, "Non-RCE1/NonRCE2/AHG5/AHG8: Adaptive Inter-Plane Prediction for RGB Content," 13th JCT-VC meeting, Incheon, Korea, document JCTVCM0230, Apr. 2013.

[25] X. Zhang, C. Gisquet, E. Francois, F. Zou, and O. C. Au, "Chroma intra prediction based on inter-channel correlation for HEVC," IEEE Trans. Image Process., vol. 23, no. 1, pp. 274-286, Jan 2014.

[26] M. Karczewicz, L. Guo, J. Sole, R. Joshi, K. Sharman, N. Saunders, and J. Gamei, "RCE2: Results of Test D1 on Rice Parameter Initialization," 15th JCT-VC meeting, Geneva, Switzerland, document JCTVC-O0239, Oct. 2013.

[27] D. Marpe, H. Kirchhoffer, V. George, P. Kauff, and T. Wiegand, "Macroblock-adaptive residual color space transforms for 4:4:4 video coding," in Image Processing, 2006 IEEE International Conference on, Atlanta, Georgia, USA, Oct. 2006.

[28] S. L. Yu and C. Chrysafis, "New intra prediction using intra-macroblock motion compensation," 3rd JVT meeting, Fairfax, Virginia, USA, document JVT-C151r1, May 2002.

[29] D.-K. Kwon and M. Budagavi, "AHG8: Video coding using intra motion compensation," 13th JCT-VC meeting, Incheon, Korea, document JCTVC-M0350, Apr. 2013.

[30] C. Pang, J. Sole, L. Guo, M. Karczewicz, and R. Joshi, "Non-RCE3: Intra motion compensation with 2-D MVs," 14th JCT-VC meeting, Vienna, Austria, document JCTVC-N0256, Jul. 2013.

[31] B. Li and J. Xu, "Non-SCCE1: Unification of intra BC and inter modes," 18th JCT-VC meeting, Sapporo, Japan, document JCTVC-R0100, Jul. 2014.

[32] C. Pang, Y.-K. Wang, V. Seregin, K. Rapaka, M. Karczewicz, X. Xu, S. Liu, S. Lei, B. Li, and J. Xu, "CE2 Test1: Intra block copy and inter signalling unification," 20th JCT-VC meeting, Geneva, Switzerland, document JCTVC-T0094, Feb. 2015.

[33] L. Guo, J. Sole, and M. Karczewicz, "Palette Mode for Screen Content Coding," 13th JCT-VC meeting, Incheon, Korea, document JCTVCM0323, Apr. 2013.

[34] P. Onno, X. Xiu, Y.-W. Huang, and R. Joshi, "Suggested combined software and text for run-based palette mode," 18th JCT-VC meeting, Sapporo, Japan, document JCTVC-M0348, Jul. 2014.

[35] Wikipedia, "Truncated binary encoding - Wikipedia, the free encyclopedia," 2014. [Online]. Available: http://en.wikipedia.org/wiki/ Truncated_binary_encoding

[36] H. S. Malvar, G. J. Sullivan, and S. Srinivasan, "Lifting-based reversible color transformations for image compression," in SPIE Applications of Digital Image Processing. International Society for Optical Engineering, August 2008. [Online]. Available: http: //research.microsoft.com/apps/pubs/default.aspx ?id=102040

[37] L. Zhang, J. Chen, J. Sole, M. Karczewicz, X. Xiu, Y. He, and Y. Ye, "SCCE5 Test 3.2.1: In-loop color-space transform," 18th JCTVC meeting, Sapporo, Japan, document JCTVC-R0147, July 2014.

[38] B. Li and J. Xu, "Fix for adaptive color space coding in JCTVC-Q0035," 17th JCT-VC meeting, Valencia, Spain, document JCTVC-Q0213, Mar. 2014.

[39] L. Zhang, J. Chen, M. Karczewicz, B. Li, and J. Xu, "Unification of colour transforms in ACT," 19th JCT-VC meeting, Strasbourg, France, document JCTVC-S0254, Oct. 2014.

[40] A. Khairat, T. Nguyen, M. Siekmann, D. Marpe, and T. Wiegand, "Adaptive cross-component prediction for 4:4:4 high efficiency video coding," in Image Processing (ICIP), 2014 IEEE International Conference on, Paris, France, Oct 2014.

[41] T. Nguyan, J. Sole, and J. Kim, "RCE1: Summary report of HEVC range extensions core experiment 1 on inter-component decorrelation methods," 14th JCT-VC meeting, Vienna, Austria, document JCTVCN0034, Jul. 2013.

[42] L. Zhang, J. Chen, J. Sole, M. Karczewicz, X. Xiu, and X. J., "Adaptive color-space transform for HEVC screen content coding," in Data Compression Conference (DCC), 2015, Snowbird, Utah, USA, Apr. 2015.

[43] P. Lai, S. Liu, and S. Lei, "AHG6: On adaptive color transform (ACT) in SCM2.0," 19th JCT-VC meeting, Strasbourg, France, document JCTVCS0100, Nov. 2014.
[44] B. Li, J. Xu, G. Sullivan, Y. Zhou, and B. Lin, "Adaptive motion vector resolution for screen content," 19th JCT-VC meeting, Strasbourg, France, document JCTVC-S0085, Oct. 2014

[45] R. Joshi, J. Xu, C. R., S. Liu, Z. Ma, and Y. Ye, "Screen Content Coding Test Model 3 Encoder Description (SCM 3)," 19th JCT-VC meeting, Strasbourg, France, document JCTVC-S1014, Oct. 2014.

[46] B. Li, J. Xu, F. Wu, X. Guo, and G. J. Sullivan, "Description of screen content coding technology proposal by Microsoft," 17th JCT-VC meeting, Valencia, Spain, document JCTVC-Q0035, Mar. 2014.

[47] W. Pu, F. Zou, V. Seregin, R. Joshi, M. Karczewicz, and J. Sole, "NonCE6: Palette parsing dependency and palette encoder improvement," 19th JCT-VC meeting, Strasbourg, France, document JCTVC-S0156, Oct. 2014.

[48] H. Yu, R. Cohen, K. Rapaka, and J. Xu, "Common test conditions for screen content coding," 20th JCT-VC meeting, Geneva, Switzerland, document JCTVC-T1015, Feb. 2015.

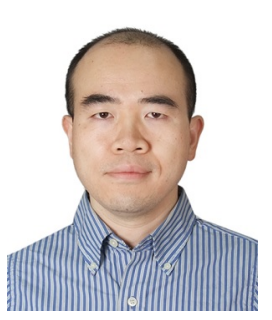

Jizheng Xu (M'07-SM'10) received the B.S. and M. S. degrees in computer science from the University of Science and Technology of China (USTC), and the Ph.D. degree in electrical engineering from Shanghai Jiaotong University, China.

He joined Microsoft Research Asia (MSRA) in 2003 and currently he is a Lead Researcher. He has authored and co-authored over 100 conference and journal refereed papers. He has over 30 U.S. patents granted or pending in image and video coding. His research interests include image and video representation, media compression, and communication. He has been an active contributor to ISO/MPEG and ITU-T video coding standards. He has over 30 technical proposals adopted by H.264/AVC, H.264/AVC scalable extension, High Efficiency Video Coding, HEVC range extension and HEVC screen content coding standards. He chaired and co-chaired the ad-hoc group of exploration on wavelet video coding in MPEG, and various technical ad-hoc groups in JCT-VC, e.g., on screen content coding, on parsing robustness, on lossless coding. He co-organized and co-chaired special sessions on scalable video coding, directional transform, high quality video coding at various conferences. He also served as special session co-chair of IEEE International Conference on Multimedia and Expo 2014.

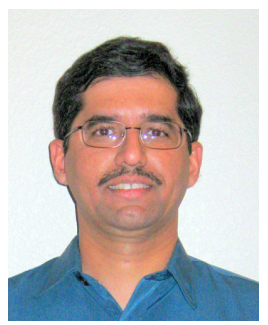

Rajan Joshi (M'95) received the the B. Tech. degree in electrical engineering and the $M$. Tech. degree in communications engineering from Indian Institute of Technology, Mumbai in 1988 and 1990, respectively, and the Ph.D. degree in electrical engineering from Washington State University, Pullman, WA, in 1996.

In 1995, he was with the Xerox Palo Alto Research Center, Palo Alto, CA. From 1996 to 2006 he was a Senior Research Scientist with Eastman Kodak Company, in Rochester, NY. From 2006 to 2008, he was a Senior Member Technical Staff with Thomson Corporate Research in Burbank, CA. He joined Qualcomm, Inc. in San Diego, CA, in 2008, where he is currently a Senior Staff Engineer/Manager. $\mathrm{He}$ has been an active participant in several past and present video and image coding standardization efforts such the Joint Collaborative Team on Video Coding (JCT-VC) for the development of the HEVC standard, and its extensions, JPEG2000, and the advanced display stream compression task group in VESA. His current research interests include video and image coding, video processing, and information theory. 


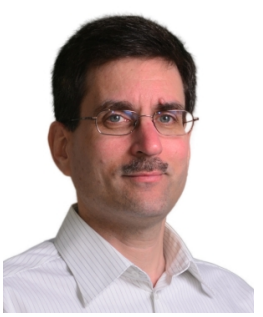

Robert A. Cohen (S'85-M'90-SM'12) received the B.S. (summa cum laude) and M.Eng. in computer and systems engineering, and in 2007 the Ph.D. degree in electrical engineering from Rensselaer Polytechnic Institute, Troy, NY. While a student, he held positions at IBM and at Harris RF Communications. From 1991 to 2001, he was a senior member of the research staff in Philips Research, Briarcliff Manor, NY, where he performed research in areas related to the Grand Alliance HDTV decoder, rapid prototyping for VLSI video systems, statistical multiplexing for digital video encoders, scalable MPEG-4 video streaming, and next-generation video surveillance systems. Since 2007 he has been a principal member of the research staff at Mitsubishi Electric Research Laboratories (MERL) in Cambridge, MA, where he performs research, publishes, and generates patents related to next-generation video coding, screen-content coding, and perceptual image/video coding and processing. $\mathrm{He}$ also actively participates in ISO/MPEG and ITU standardization activities, including chairing several ad hoc groups and core experiments, contributing to HEVC-related call for proposals and drafting the Joint Call for Proposals for coding of screen content in JCT-VC. Recently he organized the special session on screen content coding in PCS 2013, and he was a guest editor of the Signal Processing: Image Communication Special issue on advances in high dynamic range video research. His current research interests are video coding \& communications, video, image \& signal processing, and 3D point cloud compression. 